Meta

Journal des traducteurs

Translators' Journal

\title{
Gedanken zur Übersetzung poetischer Bibeltexte
}

\section{Rudolph Kassühlke}

Volume 32, numéro 1, mars 1987

La traduction biblique

Bible Translation

URI : https://id.erudit.org/iderudit/003284ar

DOI : https://doi.org/10.7202/003284ar

Aller au sommaire du numéro

\section{Éditeur(s)}

Les Presses de l'Université de Montréal

\section{ISSN}

0026-0452 (imprimé)

1492-1421 (numérique)

\section{Découvrir la revue}

\section{Citer cet article}

Kassühlke, R. (1987). Gedanken zur Übersetzung poetischer Bibeltexte. Meta, 32(1), 76-84. https://doi.org/10.7202/003284ar

\section{Résumé de l'article}

Depuis une vingtaine d'années, la science de la traduction s'est certes développée, mais, jusqu'à maintenant, elle n'a traité que de façon marginale les problèmes relatifs à la traduction poétique. Cela tient en partie au fait que l'on manque d'une définition généralement valable (non seulement dans le monde occidental, mais aussi dans les autres parties du monde) de ce qui donne à un texte son caractère poétique. Ainsi, dans le cas de l'Ancien Testament, les études concernant les passages poétiques sont contradictoires (les unes mettant l'accent sur le rythme, mais avec des conceptions qui divergent selon les spécialistes, d'autres le mettant sur le parallélisme). Le traducteur ne peut évidemment pas consulter les auteurs ou les premiers lecteurs de ces textes pour en savoir plus. Il se trouve seul face à ce qui lui apparaît comme un puzzle dont certaines pièces feraient défaut. Il en est réduit à prendre lui-même des décisions de trois ordres, en répondant aux questions suivantes : 1 . quelle était la fonction de la forme poétique du texte à traduire ? 2. quel type de traduction va-t-on adopter d'une façon générale ? 3. comment va-t-on rendre adéquatement la fonction du texte source en tenant compte du type de traduction choisi ? Par des exemples empruntés à la récente version allemande Die Gute Nachricht, dont il fut l'un des principaux traducteurs, l'auteur nous montre comment il est possible de résoudre ces questions. Dans une version mettant l'accent sur l'équivalence fonctionnelle, destinée à un usage général et non spécifiquement liturgique, il ne convenait pas de recourir à un mètre régulier, avec des rimes, comme dans la plupart des chants, pour rendre Psaumes et prières bibliques (ce qui leur aurait conféré un caractère figé et artificiel). En allemand, on a opté pour des lignes pouvant être dites d'une seule respiration (8 à 11 syllabes, au maximum 13), avec des accentuations rythmiques bien marquées. À propos du livre de Job, on s'est souvenu du caractère mnémotechnique de la poésie didactique, mais en étant conscient du fait qu 'une forme trop rigide lasserait rapidement le lecteur de trente-huit chapitres de poésie. On a donc choisi des vers blancs, sans rimes, on a recherché des métaphores et expressions idiomatiques équivalentes (mais non identiques) par rapport à celles de l'hébreu, on a usé d'allitérations et d'assonances. Pour les Proverbes, on s'est inspiré de la forme des aphorismes modernes. Dans le Cantique, les formes métriques ont varié selon les circonstances rapportées. Quant aux passages poétiques des prophètes, il ne suffit pas de les disposer en stiques pour conserver ce caractère poétique (illusion partagée par la plupart des versions modernes). Il est préférable, en raison de leur fonction particulière, de les imprimer comme de la prose, mais en donnant un caractère rythmé à cette prose et en préservant ses métaphores, jeux de mots, etc. Certains de ces passages ont la forme de la complainte, mais la fonction de l'accusation, et il ne faudra pas l'oublier dans la traduction. Un bon exemple d'emploi d'assonances, évoquant le bruit de la mer, nous est présenté à propos d'Esaïe 17.12-14. Dans une dernière remarque, l'auteur avertit ses lecteurs que les solutions valables en allemand ne sauraient être copiées servilement dans d'autres langues. Elles visent essentiellement à stimuler la réflexion, afin que chacun puisse trouver ses propres solutions, valables dans sa langue.
Ce document est protégé par la loi sur le droit d'auteur. L'utilisation des services d'Érudit (y compris la reproduction) est assujettie à sa politique d'utilisation que vous pouvez consulter en ligne.

https://apropos.erudit.org/fr/usagers/politique-dutilisation/ 


\section{GEDANKEN ZUR ÜBERSETZUNG POETISCHER BIBELTEXTE}

RUDOLF KASSÜHLKE

United Bible Societies, Siuttgart, République fédérale allemande

Résumé

Depuis une vingtaine d'années, la science de la traduction s'est certes développée, mais, jusqu'à maintenant, elle n'a traité que de façon marginale les problèmes relatifs à la traduction poétique. Cela tient en partie au fait que l'on manque d'une définition généralement valable (non seulement dans le monde occidental, mais aussi dans les autres parties du monde) de ce qui donne à un texte son caractère poétique. Ainsi, dans le cas de l'Ancien Testament, les études concernant les passages poétiques sont contradictoires (les unes mettant l'accent sur le rythme, mais avec des conceptions qui divergent selon les spécialistes, d'autres le mettant sur le parallélisme). Le traducteur ne peut évidemment pas consulter les auteurs ou les premiers lecteurs de ces textes pour en savoir plus. Il se trouve seul face à ce qui lui apparaît comme un puzzle dont certaines pièces feraient défaut. Il en est réduit à prendre lui-même des décisions de trois ordres, en répondant aux questions suivantes: 1. quelle était la fonction de la forme poétique du texte à traduire? 2. quel type de traduction va-t-on adopter d'une façon générale? 3. comment va-t-on rendre adéquatement la fonction du texte source en tenant compte du type de traduction choisi?

Par des exemples empruntés à la récente version allemande Die Gute Nachricht, dont il fut l'un des principaux traducteurs, l'auteur nous montre comment il est possible de résoudre ces questions. Dans une version mettant l'accent sur l'équivalence fonctionnelle, destinée à un usage général et non spécifiquement liturgique, il ne convenait pas de recourir à un mètre régulier, avec des rimes, comme dans la plupart des chants, pour rendre Psaumes et prières bibliques (ce qui leur aurait conféré un caractère figé et artificiel). En allemand, on a opté pour des lignes pouvant être dites d'une seule respiration $(8$ à 11 syllabes, au maximum 13), avec des accentuations rythmiques bien marquées. À propos du livre de Job, on s'est souvenu du caractère mnémotechnique de la poésie didactique, mais en étant conscient du fait qu'une forme trop rigide lasserait rapidement le lecteur de trente-huit chapitres de poésie. On a donc choisi des vers blancs, sans rimes, on a recherché des métaphores et expressions idiomatiques équivalentes (mais non identiques) par rapport à celles de l'hébreu, on a usé d'allitérations et d'assonances. Pour les Proverbes, on s'est inspiré de la forme des aphorismes modernes. Dans le Cantique, les formes métriques ont varié selon les circonstances rapportées. Quant aux passages poétiques des prophètes, il ne suffit pas de les disposer en stiques pour conserver ce caractère poétique (illusion partagée par la plupart des versions modernes). Il est préférable, en raison de leur fonction particulière, de les imprimer comme de la prose, mais en donnant un caractère rythmé à cette prose et en préservant ses métaphores, jeux de mots, etc. Certains de ces passages ont la forme de la complainte, mais la fonction de l'accusation, et il ne faudra pas l'oublier dans la traduction. Un bon exemple d'emploi d'assonances, évoquant le bruit de la mer, nous est présenté à propos d'Esaïe 17.12-14.

Dans une dernière remarque, l'auteur avertit ses lecteurs que les solutions valables en allemand ne sauraient être copiées servilement dans d'autres langues. Elles visent es- 
sentiellement à stimuler la réflexion, afin que chacun puisse trouver ses propres solutions, valables dans sa langue.

Die Publikationen zur Theorie und/oder Praxis des Übersetzens zählen mittlerweile nach Tausenden, so dass keiner mehr einen vollen Überblick haben kann. Wer aber etwas zur Problematik der Übersetzung poetischer Texte sucht, wird vor allem auf Werkstattberichte der Übersetzer selbst stossen, die sich und ihren Lesern im Nachhinein Rechenschaft über ihr Tun geben wollen. Die erst etwa zwanzig Jahre alte Übersetzungwissenschaft behandelt diese Fragen - wenn überhaupt - nur am Rand. Der Hauptgrund dafür dürfte sein, dass sie immer noch mit ihrer eigenen allgemeinen Standortsbestimmung und ihrer Abgrenzung gegenüber anderen Disziplinen beschäftigt ist. Noch schwieriger scheint es zu sein, sich darauf zu einigen, was linguistisch betrachtet eigentlich Poesie ist. Die Definitionen gehen teilweise weit auseinander. Alle haben ihre Berechtigung hinsichtlich gewisser Teilaspekte der Poesie, aber von einer allgemein akzeptablen Definition oder Theorie des Poetischen sind wir noch weit entfernt - wenn sie überhaupt je erreicht werden kann ${ }^{1}$.

Das Gesagte gilt schon für den noch relativ überschaubaren Bereich der Poesie in verwandten Sprachen, etwa der indo-europäischen, für den es zahlreiche Untersuchungen gibt. Sobald man aber diesen halbwegs vertrauten Bereich verlässt und Vergleiche mit poetischen Texten in aussereuropäischen Sprachen anstellt, fühlt man sich wirklich wie auf einem anderen Stern. Das Befremdliche liegt dann nicht so sehr an den kognitiven, überprüfbaren linguistischen Elementen wie Metaphorik, Rhythmus sowie Benutzung und Wiederholung bestimmter Lautkombinationen, als vielmehr an den emotiven Elementen, die sich der Messbarkeit immer noch weitgehend entziehen. Um in einer fremden, gar nicht verwandten Sprache fühlen zu können, braucht man ein halbes Leben, mehr als für die meisten linguistischen Untersuchungen zur Verfügung steht.

Hinsichtlich poetischer Bibeltexte, vor allem im Alten Testament, gibt es inzwischen zahllose Untersuchungen mit widersprüchlichen Ergebnissen : Die einen sprechen von der Metrik des Hebräischen, wobei die Zahl der Hebungen pro Zeile in vielen Fällen ganz unterschiedlich beurteilt wird, die anderen sprechen dem Hebräischen jegliche Metrik ab und sehen als das einzige distinktive poetische Merkmal den Aufbau in parallelen Zeilen.

Man kann sich vorstellen, dass angesichts solcher Diversität sogar eine philologische Übersetzung ${ }^{2}$ Schwierigkeiten hat bei der Wahl, wie sie die literarischen Charakteristika ihrer Vorlage adequat wiedergeben soll. Die Ưbersetzer der englischen Authorized Version von 1611 z.B. haben die Anzahl der betonten Silben je Zeile im hebräischen Text - entsprechend ihrer Analyse - mit der gleichen Anzahl betonter Silben in der Übersetzung wiedergegeben. Die New English Bible deutet die unterschiedliche Hebungszahl durch verschieden starkes Einrücken der Verszeilen an. Andere begnügen sich mit einer Druckanordnung in Verszeilen, ohne dass der Text selbst als Poesie empfunden werden könnte. Und wieder andere legen Wert auf einen gewissen Rhythmus der Übersetzung mit der Abzweckung, die Texte für die Liturgie singbar zu machen.

Für eine kommunikative Übersetzung, die Wert legt auf funktionale Äquivalenz zwischen Ausgangs- und Zieltext, wird die Entscheidung noch schwieriger, weil zusätzlich zu den Unsicherheiten im formalen und denotativen Bereich noch die Unwägbarkeiten im Bereich der Konnotationen kommen. Bei dem zeitlichen und kulturellen Abstand alttestamentlicher Texte lassen sich die Probleme, auf die der Übersetzer stösst, nicht mehr durch Rückfragen bei der ursprünglichen 
Empfängergruppe lösen. Fast ausschliessliche Instanz für alle Fragen sind die Texte selbst, und oft genug steht der Übersetzer vor einem Puzzle, von dem nicht mehr alle Teile vorhanden sind. Um die Lücken auszufüllen zu können, ist er oft genug auf seine Intuition angewiesen. Wie gefährlich das ist, braucht nicht besonders betont zu werden ; denn manchmal meint man Lücken zu sehen, wo eigentlich keine sind, und an anderen Stellen erkennt man nicht, dass etwas fehlt.

Ein Blick in irgendeinen beliebigen wissenschaftlichen Kommentar zu poetischen Bibeltexten lässt diese Schwierigkeit schnell sichtbar werden. In der Regel gibt der Verfasser zu Beginn eine "metrische" Analyse des betreffenden Abschnittes. Aufgrund der von ihm angenommenen Metrik glaubt er dann feststellen zu können, wo aus "metrischen" Gründen ein Wort, eine Zeile auszuscheiden ist, etwas das irgendein Redaktor später aus irgendwelchen Gründen in den Text eingefügt haben muss. Nicht ganz so häufig wird auch vermerkt, dass aus "metrischen" Gründen vielleicht ein Wort fortgelassen wurde, eine Beobachtung, die man bei Liedern, auch in nichteuropäischen Sprachen, ständig machen $\mathrm{kann}^{3}$. Trotz dieser Analyse wird dann im anschliessenden Erklärungsteil der Text so kommentiert, als sei er ein reiner informativer Prosatext ohne jegliche Informationslücke.

Der Übersetzer steht angesichts dieser wissenschaftlichen Situation vor Entscheidungen, die notgedrungen subjektiv ausfallen müssen. Erstens muss er eine Antwort auf die Frage finden, welche Funktion die poetische Form des jeweils zu übersetzenden Textes hat. Zweitens ist es die Frage nach dem Übersetzungstyp, die er entscheiden muss. Und drittens muss er entscheiden, wie er die erkannte Funktion im gewählten Übersetzungstyp adequat wiedergeben will.

Die folgenden Ausführungen sollen deutlich machen, wie hinsichtlich dieser Fragen bei der Arbeit an der "Bibel in heutigem Deutsch (Die Gute Nachricht)" verfahren und entschieden wurde.

\section{PSALMEN UND GEBETE}

Im Gottesdienst Israels wurden Psalmen und Gebete in der Regel gesungen, wie man an den Regiebemerkungen zu Beginn vieler solcher Texte sehen kann. Das schliesst nicht aus, dass sie für den "Hausgebrauch" auch als Gebete rezitiert wurden. Bei einer kommunikativen Übersetzung wie der "Bibel in heutigem Deutsch", die keine kirchlich-offizielle Funktion hat und auch nicht haben will, wird der "Hausgebrauch" vorausgesetzt. Metrisch gebundene Form, d.h. eine solche, die ein festes Versmass verwendet, wie es bei Liedern üblich ist, kommt für Gebete nicht in Betracht. Grund dafür ist, dass diese Form im Deutschen die Konnotation des Fiktiven hat und damit dem Grundgedanken des Gebetes widerspräche. Andererseits sind Psalmen keine spontan formulierten Gebete; sie zeigen deutlich, dass sie immer wieder an neue Situationen angepasst worden sind. Die Übersetzung kommt aufgrund dieser überlegungen zu folgendem Muster :

Die einzelnen Zeilen sollen mit einem Atemzug laut ausgesprochen werden können; deshalb sollen sie, von ganz seltenen Ausnahmen abgesehen, acht bis elf (maximal dreizehn) Silben haben. Um rhythmisches Sprechen zu ermöglichen, sollten nie zwei betonte Silben direkt aufeinander folgen, aber auch nicht mehr als drei unbetonte, weil der Leser sonst verunsichert wird und an den falschen Stellen betont, was inhaltlich sekundäre Elemente in den Vordergrund bringen könnte. Wenn sich beim Übersetzen unbeabsichtigt ein festes Versmass einstellt, muss man spätestens nach sechs bis sieben Zeilen diesen Rhythmus unterbrechen, weil er sonst nach Fortsetzung 
bis zum Ende verlangt. Im nachfolgenden Beispiel werden die bei natürlichem Lesen betonten Silben unterstrichen :

Herr!

Hast du mich für immer vergessen?

Wie lange willst du dich denn noch verbergen?

Wie lange sollen mich die Sorgen quälen,

der Kummer Tag für $\mathrm{T} a \mathrm{~g}$ an meinem Herzen nagen ?

Wie lange dürfen mich die Feinde noch bedrängen ?

Sieh mich doch wieder an, Herr!

Gib mir doch Antwort, du mein Gott!

Mach es wieder hell vor meinen Augen,

damit ich nicht in Todesnacht versinke!

Sonst sagen meine Feinde : "Den haben wir erledigt !" (!)

und jubeln über meinen Sturz.

Doch ich verlasse mich auf deine Liebe,

ich juble über deine Hilfe. Mit meinem Lied will dir danken, Herr,

weil $\mathrm{d} u$ so $\mathrm{g} u \mathrm{t} \mathrm{zu}$ mir gewesen bist.

(Psalm 13)

DAS BUCH IJOB (HIOB)

Nach Meinung von Kennern gehört dieses biblische Buch zu den schönsten poetischen Stücken der Weltliteratur. Leider wird das in der Übersetzung nur selten deutlich, weil man im Bestreben, eine philologisch exakte Übersetzung zu bieten, gar nicht nach einer möglichen poetischen Form in der Zielsprache fragt ${ }^{4}$. Das Ergebnis ist ein Prosatext, der durch seine Widerholungen sehr schnell ermüdend wirkt ${ }^{5}$. Der heutige Leser wünscht sich die Information möglichst kompakt, es sei denn, sie würde ihm in einer Form geboten, die zum geniessenden Lesen einlädt. Die Frage nach der Funktion der Poesie im Buch Ijob ist darum vordringlich.

Auf den ersten Blick könnte man das Buch für die ergreifende Schilderung eines Mannes halten, der in seinem Leben eine Kette von schweren Leiden durchstehen musste. Dann wäre eine Übersetzung in metaphernreicher rhythmischer Sprache ähnlich wie bei den Psalmen - die angemessene Form. Das Buch Ijob ist aber ein gewaltiges Lehrgedicht, das zur Gattung der sogenannten biblischen "Weisheitsliteratur" gehört. Der Verfasser setzt sich mit einer Reihe von theologischen Fragen seiner Zeit auseinander, die ihm wohl persönlich arg zu schaffen machten. Mit seinen Argumenten will er sein Wissen an seine Schüler weitergeben. Zieht man in Betracht, dass Bücher damals fast unerschwinglich teuer waren und darum das menschliche Gedächtnis den gesamten Lernstoff speichern musste, so liegt der Schluss nahe, dass, die poetische Form die Memorierbarkeit erleichtern sollte. Herbert V. Klem (1982) untersucht das Hör- und Lernverhalten einer Gesellschaft, die nicht auf schriftliche, sondern auf mündliche Kommunikation aufgebaut ist, und kommt zu dem einleuchtenden Ergebnis, dass für die gesamte Gesellschaft wichtige Kommunikation in poetischer Form weitergegeben wird. Das zeigt er nicht nur an Texten des YorubaVolkes in Südostnigerien, sondern auch an Evangelientexten, die ins Aramäische zurückübersetzt worden waren ${ }^{6}$.

Auch wenn eine moderne deutsche Übersetzung das Buch Ijob nicht als Memorierstoff behandeln will, liegt die Frage nahe, ob nicht eine angemessene poetische Form den heutigen Leser eher dazu bewegen kann, das Buch einmal ganz zu lesen. Freie Rhythmen nach Art moderner Lyrik wären vorstellbar, wenn man Einzelabschnitte dieses Buches wiedergeben wollte. Bei einem Lehrgedicht von 38 
Kapiteln bekäme jeder Einzelabschnitt aber so viel Gewicht, dass der Leser sich wieder daran festbeissen und den Zusammenhang aus den Augen verlieren würde. Die Übersetzer haben sich deshalb für eine leicht veraltete poetische Form, den Blankvers, entschieden, um einen sehr alten Text wiederzugeben. Der füfhebige Jambus ohne Endreim entspricht von allen Versmassen am ehesten dem deutschen Sprechrhythmus; viele können ihn überlesen, ohne es zu merken. Die Übersetzung gebraucht daneben immer wieder anstelle hebräischer Idiome und Metaphern gleichbedeutende deutsche.

Von dieser Art hab ich genug gehört !

Nur Last ist euer Trost für mich, nicht Hilfe!

Machst du nun endlich Schluss mit dem Gerede ?

Was zwingt dich denn, mir ständig zu erwidern?

Wenn ihr jetzt hier an meiner Stelle wärt,

dann könnte ich genau dasselbe sagen.

Ich könnte meinen Kopf sehr weise schütteln

und euch mit schönen Sprüchen reich bedenken.

Ich gäb euch neue Kraft - mit meinem Mund ;

ich zeigte euch mein Mitleid - mit den Lippen.

(Ijob 16,2-5)

Es versteht sich von selbst, dass bei der Ungleichheit von Ausgangs- und Zielsprache solch eine feste Form eine "wörtliche" Übersetzung unmöglich macht. Manchmal kann man mit der Wahl eines synonymen Wortes dem Versmass gerecht werden. Wenn aber das Versmass in einer Zeile mehr Silben braucht als die "exakte" Übersetzung hergeben kann, muss man die Zeile notgedrungen auffüllen. Im umgekehrten Fall lässt sich unter Umständen ein Wort streichen, das für ein richtiges Verstehen des Textes nicht erforderlich ist. Und wenn sich die Übersetzung einer Zeile nicht in einer einzelnen Zeile unterbringen lässt, dann muss man ihn in zwei Zeilen giessen. Die Übersetzung soll ja nicht die poetische Form des Ausgangstextes widerspiegeln, sondern dessen Inhalt in einer Form ausdrücken, die der Zielsprache angemessen ist.

Ein altes Stilmittel, das in allen germanischen Sprachen bekannt ist, ist der Gebrauch von Alliteration und Assonanz. Die vorliegende Übersetzung benutzt beides, wenn es sich auf natürliche Weise ergibt :

Wenn jemand meinen Kummer wiegen wollte und meine Leiden auf die Waage legte sie wären schwerer als der Sand am Meer.

Was Wunder, wenn ich wirre Reden fuhre?

Die Pfeile Gottes haben mich getroffen und meinen Geist mit ihrem Gift verstört.

$\cdots$

Kein Esel schreit auf saftig grüner Weide, und jeder Stier ist still, hat er sein Futter.

Doch wer mag ungesalzne Speisen essen?

Wem schmeckt der weisse Schleim von einem Ei?

(Ijob 6,2-6)

\section{SPRICHWÖRTER}

Das Buch der Sprichwörter gehört zwar auch zur "Weisheitsliteratur", enthält aber zum weitaus überwiegenden Teil Einzelsprüche, im Hebräischen in zwei parallelen Zeilen ausgeformt. Diese Form hat - darin sind sich die Exegeten weitgehend einig ebenfalls eine mnemotechnische Funktion. Ähnlich wie deutsche Bauernregeln über Wetter und Jahreszeit drücken sie alte Volksweisheit aus. Die gereimte Form deutscher Bauernregeln würde aber den biblischen Sprichwörtern ihren ernsthaften Lehrcharakter nehmen. Deshalb benutzt die Übersetzung die Form moderner 
Aphorismen und als Stilmittel neben den schon genannten lautlichen Elementen Wortspiele, Gegensätze und die Ausnutzung der Polysemie bestimmter Wörter. Die folgenden Beispiele sind alle dem Buch der Sprichwörter (Sprüche) entnommen :

Wer geradlinig lebt, lebt ohne Angst ; wer krumme Wege geht, wird irgendwann ertappt. $(10,9)$

Lass einen Faulen für dich arbeiten; das wird dir so wohltun wie Essig den Zähnen und

Rauch den Augen. $(10,26)$

Nicht angesehen sein und einen Diener haben ist besser als berïhmt sein und nichts zu essen haben. $(12,9)$

Wer sich auf Abwege begibt, bekommt den Lohn dafür, aber der Lohn guter Menschen ist besser. $(14,14)$

Auf Stolz folgt Sturz, nach übermut kommt Untergang. $(16,18)$

\section{DAS HOHELIED}

Das Hohelied, eine Sammlung von Liebesliedern, wie man sie auch aus anderen Kulturen des Alten Orients kennt, stellt den Übersetzer vor ganz andere Probleme. Die äusserst blumenreiche orientalische Ausdrucksweise ist für mitteleuropäisches Empfinden in den Gefühlskomponenten zu warm und schwülstig. Um als Liebeslieder empfunden zu werden, müssen die Texte in Form und Ausdruck eine schlichte, anspruchslose Schönheit aufweisen. Den Charakter einer Sammlung von Liedern drückt die Übersetzung durch die Verwendung verschiedener Versmasse aus. Als Verständnishilfe wird der jeweilige Sprecher (oder Sänger) genannt. Nach den Texten steht das verwendete Versmass :

SIE Sag mir, Geliebter, wo kann ich dich finden?

Wo ruhn deine Schafe mittags, wenn's heiss wird?

Andere Hirten, was sollen sie denken,

wenn ich nach dir frage, überall suche?

ER Musst du mich fragen, du Schönste der Frauen?

Du musst es doch wissen, wo du mich findest!

Nimm deine Zicklein und folge dem Schafsweg!

Dort wirst du mich treffen, nah bei den Zelten.

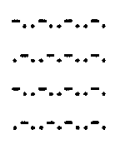

Für die Beschreibung von Personen werden oft Trochäen benutzt :

ER Schön wie Tirza bist du, Freundin,

strahlend wie Jerusalem;

wie ein Trugbild in der Wüste

raubt dein Anblick mir den Atem.

Wende deine Augen von mir,

denn sie halten mich gefangen.

Ereignisse werden in der Regel in Jamben ausgedrückt :

SIE Nachts lieg ich auf dem Bett und kann nicht schlafen.

Ich sehne mich nach ihm und suche ihn,

doch nirgends kann mein Herz den Liebsten finden.

Ich seh mich aufstehn und die Stadt durcheilen, 
durch Gassen streifen, über leere Plätze ich sehne mich nach ihm und suche ihn, doch nirgends kann ich meinen Liebsten finden.

Die Wache greift mich auf bei ihrem Rundgang.

"Wo ist mein Liebster, habt ihr ihn gesehn ?"

Nur ein paar Schritte weiter find ich ihn.

Ich halt ihn fest und lass ihn nicht mehr los; ich nehm ihn mit nach Hause in die Kammer, wo meine Mutter mich geboren hat.

Ihr Mädchen von Jerusalem, lasst uns allein !

Denkt an die scheuen Rehe und Gazellen :

Wir lieben uns, schreckt uns nicht auf!

\section{AUSSPRÜCHE DER PROPHETEN}

Alle formalen Beobachtungen, die man an Psalmen, Liedern und Weisheitssprüchen in der hebräischen Bibel machen kann, treffen auch für die meisten Texte der Prophetenbücher zu. Darum bieten die meisten Übersetzungen diese Texte in stichischem Druck dar. Das macht aber aus den Zeilen nicht automatisch Poesie. Meistens sind die Übersetzungen wegen ihres Bestrebens um wörtliche Wiedergabe rhythmisch unbefriedigend und inhaltlich schwierig. Metaphern und idiomatische Wendungen wirken in dieser Wiedergabe fremdartig, oft genug sind sie unverständlich.

Die vielleicht wichtigste Frage ist aber mit diesen Überlegungen noch gar nicht angeschnitten : Welche Funktion hatte die poetische Form der Prophetensprüche in der Ausgangskultur? Nach Abwägen aller exegetischen Erkenntnisse kann man wohl sagen, dass diese relativ gebundene Form im Alten Orient den Botschaften einen besonderen Nachdruck verlieh. Wer längere Zeit Kontakt zu einer Kultur mit vorwiegend mündlicher Kommunikation gehabt hat, ist auch nicht mehr erstaunt uiber ad hoc formulierte Poesie. Viele Übersetzer lassen sich anscheinend von der exegetischen Feststellung, dieser oder jener prophetischer Text habe die Form eines Klageliedes oder einer Totenklage, dazu verführen, ihn nun auch so zu übersetzen. Inhaltlich sind solche Texte aber meistens keine Klagelieder, sondern massive Anklagen, mit denen der Prophet im Auftrag Gottes geschehenes Unrecht brandmarkt. Solchen Texten den formalen Anschein von Poesie zu geben, muss im Grunde schon den Leser irritieren, sie mit einem festen Versmass als "Klagelieder" wiederzugeben, würde sie zwar sehr flüssig lesbar machen, ihnen aber gleichzeitig allen anklagenden Ernst nehmen. Im Deutschen ist darum wahrscheinlich für diese Art von Prophetensprüchen ein Druck wie für Prosatexte besser angebracht. Die Sprache selbst sollte die einer konzentrierten, rhythmischen Prosa sein, die sich von erzählender Prosa durch Prägnanz, Metaphernreichtum und gezielten Gebrauch von Ironie, Satire und Wortspielen unterscheidet. Den folgenden Ausschnitt aus einem der sogenannten "Klagelieder" könnte man ohne Schwierigkeiten stichisch drucken, die "parallelen Zeilen" des hebräischen Textes kann man unschwer erkennen :

Wie konnte sie solch eine Hure werden, die Stadt, die früher so treu war ? Einst herrschte in ihr das Recht, ihre Bewohner folgten Gottes Geboten ; jetzt aber wohnen dort lauter Mörder ! Jerusalem, früher warst du wie reines Silber, jetzt aber bist du nichts als silbrigglänzende Schlacke! Früher warst du wie guter Wein, jetzt aber ist der Wein verwässert ! Deine Führer - Aufrührer sind sie, die mit Dieben unter einer Decke stecken, scharf auf Geschenke und Bestechungsgeld ! Aber den Waisen verhelfen sie nicht zu ihrem Recht, und die Klagen der Witwen hören sie gar nicht erst an...

(Jesaja 1,21-23)

Anders verhält es sich mit Gebeten und Zukunftsvisionen in den Prophetenbüchern. Für sie gilt das für die Übersetzung der Psalmen Gesagte. Der 
folgende Abschnitt ist im Hebräischen voll von Lauten, die den Ansturm des Meeres "hören" lassen. Die Übersetzung versucht, das auch im Deutschen durch Assonanzen und Alliterationen zu erzielen :

Hört ! Völkermassen brausen heran,

sie tosen wie das rauschende Meer!

Nationen sind in Aufruhr,

sie toben wie das aufgewühlte Meer!

Doch Gott bedroht sie, und sie weichen zurück

wie Spreu, die der Wind auf den Bergen davonbläst,

wie trockene, ausgerissene Disteln,

die der Sturm übers Land wirbelt.

Am Abend noch herrscht helles Entsetzen -

bevor der Morgen graut, ist alles vorbei!

So geht es denen, die uns berauben wollen;

das ist das Schicksal aller, die zum Plündern kommen!

(Jesaja 17,12-14)

Bei ausgesprochenen Spottliedern sollten keine Bedenken bestehen, festes Versmass, eventuell auch Reim zu verwenden, wie bei dem Spottlied auf den König von Babylon :

Man schreckt die Könige der Völker hoch,

sie alle springen auf von ihren Thronen.

Da stehen sie und heissen dich willkommen,

der Chor der Schatten ruft dir spöttisch zu :

"Sieh da, nun bist auch du für immer hier,

ganz ohne Macht, genauso schwach wie wir!"

Dahin ist nun die Pracht, die dich umgab,

dahin die rauschende Musik der Harfen,

hinunter in die dunkle Totenwelt.

Das Bett, auf dem du liegen darfst, sind Maden,

und Würmer sind die Decke über dir.

(Jesaja 14,9b-11)

\section{NACHBEMERKUNG}

Was die Übersetzer der "Bibel in heutigem Deutsch" unternommen haben, kann sicher angefochten und hinterfragt werden. Es sollte auf keinen Fall in einer anderen Sprache kopiert werden, weil jede Sprach- und Kulturgruppe andere Empfindungen mit poetischen Formen verbindet. Diese "Gedanken zur Übersetzung poetischer Bibeltexte" möchten zum Nach-denken anregen und zum Suchen nach eigenen, angemessenen Lösungen.

\section{Notes}

1. Vgl. Robert de Beaugrande 1978, S. 23 : "It is also inadvisable to claim that the relation of poetic language to ordinary language can be settled for once and for all. Some researchers (such as Bierwisch 1965) consider poetic language as secondary and derivative from ordinary language. Some (such as Jokobson 1960) see the two as alternate varieties of the total language. Still others (such as Coseriu 1971) view poetic language as the realization of the total potential of a language, and ordinary language as a reduction of that potential" Bei de Beaugrande ist auch eine umfassendere Bibliographie zu den Fragen der Poesie und ihrer Übersetzung zu finden.

2. Zur Unterscheidung der verschiedenen Übersetzungstypen s. Katharina Reiss, Paraphrase und Übersetzung, Versuch einer Klärung, in Gnilka/Rüger 1985, S. 273-278.

3. Bei der Analyse von alten Gesängen in Ful (Fulani, Peul), einer Sprache Westafrikas, traf ich wiederholt auf dieses Problem. Von drei gebildeten einheimischen Informanten erhielt ich nicht selten drei verschiedene Interpretationen derselben Textstelle. Bei Gesprächen mit südafrikanischen Übersetzern ergab sich das gleiche Bild auch für ihre Sprachen. Je weiter der Text zeitlich zurückliegt, desto unsicherer wird das Verständnis. 
4. Eine Ausnahme im Deutschen ist der Kommentar von Helmut Lamparter (s. Bibliographie), der für die Übersetzung ein festes Versmass verwendet und diese Entscheidung - fast mit einem schlechten wissenschaftlichen Gewissen - im Vorwort verteidigt.

5. Bei Nachfragen in verschieden zusammengesetzten Gruppen in der Bundesrepublik Deutschland zeigte sich, dass bei denen, die dieses Buch zu lesen angefangen hatten, im Durchschnitt $90 \%$ nach 13-14 Kapiteln aufgaben, weil ihnen die Argumentation zu langatmig wurde.

6. Ähnliche Beobachtungen haben mir Missionare von verschiedenen tschadohamitischen Völkern in Nordkamerun und im Tschad mitgeteilt.

\section{BIBLIOGRAPHIE}

Die Bibel in heutigem Deutsch, Die Gute Nachricht des Alten und Neuen Testaments, Deutsche Bibelgesellschaft, Stuttgart, 1982.

DE BEAUGRANDE, Robert (1978) : Factors in a Theory of Poetic Translating, Assen, The Netherlands, Van Gorcum.

BIERWISCH, Manfred (1965) : "Poetik und Linguistik", in : H. Kreuzer \& R. Gunzenhäuser (eds.), Mathematik und Dichtung, Versuche zur Frage einer exakten Literaturwissenschaft, München, Nymphenburger.

COSERIU,, Eugenio (1971) : Thesen zum Thema "Sprache und Dichtung", in : W.D. Stempel (ed.), Beiträge zur Textlinguistik, München, Fink.

GNILKA, Joachin \& Hans Peter RÜGER (eds.) (1985) : Die Übersetzung der Bibel, Aufgabe der Theologie, Stuttgarter Symposion 1984, Bielefeld, Luther-Verlag.

JAKOBSON, Roman (1960) : "Linguistics and Poetics", in : Sebeok (ed.), Style in Language, Cambridge, MIT.

KASSÜHLKE, Rudolf (1984) : "Die Sprache der Bibelübersetzung zwischen Tradition und Mission", in : Mönig (ed.), Sprechend nach Worten suchen, Probleme der philosophischen, dichterischen und religiösen Sprache der Gegenwart, München-Zürich, Schnell \& Steiner.

KLEM, Herbert V. (1982) : Oral Communication of the Scripture, Insights from African Oral Art, Pasadena, William Cary Library.

LAMPARTER, Helmut (1955) : Das Buch der Anfechtung (Das Buch Hiob), Aus : "Die Botschaft des Alten Testaments", Bd. 13, Stuttgart, Calwer Verlag, 2.Aufl. 